\title{
Comportamiento espacial de la corrupción en México a nivel entidad federativa 2001-2010
}

\author{
Spatial behavior of corruption in Mexico at state \\ level 200I-2010
}

doi: http://dx.doi.org/10.32870/

espiral.v24i70.3997

Ignacio Javier Cruz Rodríguez

\section{Resumen}

Este artículo explora la dimensión espacial del índice de corrupción a nivel entidad federativa en México entre el año 2001 y 2010. Se muestra que el patrón de localización de la corrupción no es aleatorio y que la aglomeración de entidades con altos valores se encuentra en el centro del país. Se utilizan como determinantes de la corrupción el producto interno bruto y el nivel de intervención del Gobierno. La interpretación de los resultados muestra que, para la mayoría de los años analizados, existe efecto de desbordamiento de la corrupción, y que el nivel de actividad económica está relacionado positivamente con la corrupción. También se discuten las implicaciones de la política de combate a la corrupción.

Palabras clave: corrupción, Gobierno estatal, análisis espacial, México, contagio.

\begin{abstract}
This paper explores the spatial distribution of corruption index at state level in Mexico between 200I and 2010. The corruption index shows a non-random pattern and is detected that the cluster of states is located at country center. It's used GDP as determinant of corruption index, the level government intervention and gender issues. The results show that there is a spillover effect for corruption among states in almost all years. There is a positive relation between GDP and corruption. Policy implications are discussed.
\end{abstract}

Keywords: corruption, state government, spatial analysis, Mexico, contagion.

- Profesor-Investigador del Centro de Investigaciones Socioeconómicas de la Universidad Autónoma de Coahuila (Cátedras CONACYT), México. ijcruzro@conacyt.mx Fecha de recepción: 24 de septiembre de 2015. Fecha de aceptación: 20 de marzo de 2017. 


\section{Introducción'}

La corrupción es un problema que tiene la atención de Gobiernos, organismos nacionales e internacionales, ciudadanos y académicos. Sus causas y efectos son materia de una vasta literatura.

Los estudios sobre determinantes de la corrupción, en su gran mayoría, toman datos a nivel país, por lo que son escasos los análisis que toman en cuenta información desagregada para regiones al interior de las naciones. Resulta de gran importancia considerar que la corrupción puede tener diferentes niveles al interior de un país, es decir, que existan regiones en las que la corrupción sea alta o baja. Las diferencias posibles entre regiones permiten preguntarse si la distribución de la corrupción a través de las diferentes regiones sigue un patrón particular o si está distribuida aleatoriamente.

Un patrón espacial puede interpretarse como la existencia de un efecto de desbordamiento de un fenómeno a través de regiones. Este efecto indica que la variable de interés puede afectar a la misma en otra región. Es decir, en un patrón de este tipo, la variable de interés se disemina de una región a otra (Becker, Egger y Siedel, 2009, p. 10).

En este trabajo se busca responder a la pregunta "¿Las entidades federativas que han exhibido altos niveles de corrupción han tendido a aglomerarse espacialmente cerca de otras entidades que han mostrado altos niveles de corrupción, y viceversa?". Además, se busca estimar el efecto de variables consideradas por la literatura como determinantes.

Para ello, este trabajo se divide en cinco secciones. En la primera, se estudia el concepto y el contexto de la corrupción 
en México. En la segunda, se presenta la evidencia empírica internacional respecto de los determinantes de la corrupción, incluida la variable espacial. En la tercera sección, se presentan los hechos estilizados sobre los índices de corrupción en México y los datos comparativos nacionales e internacionales que sirven para dimensionar los niveles de corrupción de México. En la cuarta, se presenta el análisis econométrico que determina la relevancia del análisis espacial y de los otros determinantes. Finalmente, se muestran las conclusiones y las recomendaciones de política derivadas de los hallazgos.

\section{Concepto, clasificación y contexto de la corrupción en México}

Existen diversos conceptos de corrupción en la literatura. Uno de las más comunes es el acuñado por Huntington (1972, p. 63), quien establece que la corrupción es una desviación en el comportamiento de un funcionario público, que se aparta de las reglas establecidas para ponerse al servicio de intereses privados. Otro concepto, parecido al anterior, es el de la organización Transparencia Internacional, quien afirma que la corrupción es el abuso del poder público para beneficio privado (Transparencia Internacional, 2015). Ambos conceptos refieren explícitamente a las acciones que lleva a cabo un servidor público. Una definición de corrupción que sobrepasa la acción de actores públicos es la siguiente: el desvío del criterio que debe orientar la conducta de un tomador de decisiones a cambio de una recompensa, o de una persona en relación a sus obligaciones como ciudadano (Casar, 2015, p. 9).

La corrupción se presenta en distintos círculos, como los espacios públicos, privados y sociales. La corrupción en los ámbitos públicos tiene relación con el aparato del Estado, servidores públicos y aquellas entidades que son 
fundamentales para entender la vida del Estado, como los partidos, sindicatos, organizaciones empresariales y medios de comunicación electrónica. La corrupción privada se genera en las empresas, en la multiplicidad de relaciones comerciales y financieras entre ellas. Por lo que toca a la corrupción social, esta se gesta al interior de las organizaciones no gubernamentales, o en otras varias instancias del tejido social (Cárdenas y Mijangos, 2005).

Las mismas esferas mencionadas en el párrafo anterior, agregando la corrupción relacionada con el narcotráfico y el crimen organizado, son estudiadas por Martínez (2004). Según el autor, la corrupción de la esfera oficial tiene diversas aristas, por ejemplo, cuando los funcionarios obtienen dinero por aplicar la ley, por utilizar información privilegiada, por asignar puestos a amigos o familiares, por corromperse en procesos de nacionalización o privatización, o cuando se asocian con líderes sociales y sindicales.

En cuanto a la corrupción privada, Martínez (2004) indica que esta comprende variados mecanismos de evasión fiscal, la que sucede en los mercados financieros y de valores o la que proviene del narcotráfico y el crimen organizado (apreciable en el lavado de dinero y el pago de protección a las fuerzas de seguridad pública o al Ejército).

En cuanto a la corrupción social, esta se puede apreciar en situaciones en las que los ciudadanos pretenden obtener beneficios sin cumplir con algún requisito para obtenerlos.

Por otra parte, es necesario destacar la forma en que fenómenos como el narcotráfico, presente en la vida políticosocial de México pueden afectar a la corrupción y contribuir al debilitamiento de las instituciones. De acuerdo con Casar (2015, p. 22), los países que presentan los niveles más altos de corrupción son aquellos que tienen instituciones políticas y jurídicas más débiles y con menor aceptación.

De acuerdo con Thoumi (1999), la relación entre narcotráfico y corrupción puede expresarse de diversas maneras: 1)

\section{6}


por la debilitación del sistema judicial; 2) por su influencia en el Poder Legislativo, que lleva a la derogación de leyes; 3) por la utilización del sector financiero en operaciones de lavado de dinero; 4) por la vulnerabilidad de la Policía y el Ejército, que pueden participar en la formación de guerrillas; 5) por su contribución a la formación de expectativas de enriquecimiento rápido en la población y el debilitamiento de las restricciones morales necesarias para que los ciudadanos respeten las leyes de propiedad, lo que puede terminar por incentivar el fraude y el robo. Thoumi (1999) indica que la presencia de narcotráfico es un disparador tanto del crecimiento de la corrupción como de su propagación, pero reconoce que ambos fenómenos se alimentan entre sí.

Un caso empírico que relaciona corrupción y narcotráfico es Colombia en los años ochenta. De acuerdo con Lee III y Thoumi (1998), los siguientes factores se combinaron en dicho país para generar el desarrollo de la industria de las drogas, y con ello el crecimiento de la corrupción:

[...] la deslegitimación del régimen, la debilidad de la sociedad civil, la gran propensión a resolver disputas a través de la violencia, la geografía del país, la estructura del sistema político y partidario, obstáculos a la movilidad social, el gran tamaño de las actividades económicas ilegales y la aceptación del contrabando y el lavado de dinero.

Muchos de los problemas mencionados en el párrafo anterior existen en México. Se pueden citar tres ejemplos: primero, la geografía del país implica la cercanía con el mercado consumidor de drogas más grande del mundo, lo cual ha convertido a México en territorio para el trasiego de las mismas. Segundo, el gran tamaño de las actividades económicas ilegales -entre las que se encuentran algunas asociadas al narcotráfico, como el secuestro, y otras actividades como la economía informal-, en conjunto, representan el $23.7 \%$ del producto interno bruto (PIB), de acuerdo con el 
INEGI (2014), es decir, casi la cuarta parte de la economía nacional. Tercero, la movilidad social en México presenta serios problemas, ya que, según un estudio del Centro de Estudios Espinosa Yglesias, cuarenta y ocho de cada cien mexicanos que se encuentran en el quintil más bajo de la distribución del ingreso se mantienen ahí (CEEY, 2013). Con estos datos, y dada la evidencia empírica mencionada, puede ser más fácil entender la relación entre narcotráfico y corrupción en México.

\section{Literatura relacionada}

La literatura relacionada con la corrupción indica claramente sus posibles impactos. Uno de los más importantes, y que ha sido ampliamente documentado, es el que tiene sobre la economía.

Soto (2003) menciona que la primera afectación de la corrupción sobre la economía se da en las decisiones de producción y el esfuerzo productivo de las empresas, pues por la corrupción se reducen al mismo tiempo la eficiencia económica y la productividad.

Otra variable de gran importancia que se ve impactada negativamente por la corrupción es la inversión. Con una muestra de sesenta y ocho países, Mauro (1995) señala la existencia de una fuerte relación negativa entre corrupción e inversión doméstica. Kauffman y Wei (1999), por su parte, hallan evidencia de que la corrupción disminuye el flujo de inversión extranjera directa. Estos autores calculan que el retorno de las inversiones puede disminuir hasta un 20\% como consecuencia de la presencia de corrupción en una economía.

Una variable de gran importancia que se ve reducida por la corrupción es el crecimiento económico. No obstante, de acuerdo con Hodge, et al. (2009), este impacto es menor en países con bajos niveles de regulación. Otras variables que 
la corrupción puede afectar son la estructura de mercado (Leff, 1964) y los costos de hacer negocios (Kauffman y Wei, 1999). Los impactos económicos no son los únicos: según Cárdenas y Mijangos (2005), otras consecuencias que tiene la corrupción en un país son que socava la legitimidad de las instituciones públicas y atenta contra la sociedad, el orden moral y la justicia, así como contra el desarrollo integral de los pueblos. Los estudios mencionados enfatizan la corrupción en general pues, como se verá más adelante, hay diversas formas y esferas en las que la corrupción se lleva a cabo. No obstante, es necesario mencionar que dichos estudios utilizan la misma forma de cuantificar la corrupción.

Los estudios sobre determinantes de la corrupción han mostrado diversas variables que la impactan. Según Chaudrhy y Shabbir (2007), las variables de tipo económico parecen tener un mayor impacto que otras. No obstante, la exploración espacial de la corrupción ha recibido poca atención, y los estudios existentes toman la información a nivel país, con lo cual se omiten las posibles diferencias en los niveles de corrupción en diferentes regiones al interior de los mismos.

El nivel de producción de una economía es considerado comúnmente en los estudios que buscan determinantes de la corrupción. ${ }^{2}$ La base teórica para estudiar la relación entre producción y corrupción parte de la existencia de escasez de recursos en cualquier economía. Esta situación puede hacer que la corrupción sea un mecanismo para obtener de manera rápida ingresos adicionales en aquellos territorios donde hay un bajo nivel de producción (además de que existe debilidad institucional implicada), por lo que la corrupción es menor en territorios en los que la escasez no es tan grave.

2. Se puede incluir el nivel de educación en lugar del nivel de producción. No obstante, dada la estrecha relación entre educación y producción, es altamente posible que incluir ambas variables genere un problema de colinealidad. En todo caso, una puede ser variable proxy de la otra. 
Esto significa que, desde esta perspectiva, la relación entre producción y corrupción es negativa. Al respecto, la literatura reporta estudios como los de Treisman (2000), Kunicova y Rose-Ackerman (2005), Lederman, Loayza y Soares (2005), Serra (2006), Aman Ullah y Eatzaz (2006), Chang y Golden (2007), Florino y Galli (2010) y Goel y Ram (2013), en cuyos hallazgos encuentran una relación negativa entre el nivel de corrupción y producción por habitante. Todos los estudios mencionados se presentan a nivel país y ninguno presenta datos desagregados.

En contraste, existe la hipótesis de que la corrupción es cíclica, conocida también como la "hipótesis Kindleberg". Esta hipótesis enfatiza que los estándares morales de la sociedad disminuyen cuando hay un fuerte crecimiento económico, implicando que la codicia se convierte en el motor de las decisiones económicas. Si este es el caso, la relación entre economía y corrupción será positiva, pues en lugares con mayor actividad económica habrá más corrupción que en aquellos con menor nivel de actividad. La evidencia que reporta tal relación se puede encontrar en Paldam (2002), Braun y Di Tella (2004), Frechette (2006) y Ata y Arvas (2011).

Por otro lado, uno de los primeros estudios en explorar la dimensión espacial de la corrupción fue el de Becker, Egger y Siedel (2009), quienes interpretan como contagio el patrón espacial que encuentran. Su estudio tiene como unidades de observación a ciento veintitrés naciones. Los autores hallan un patrón de autocorrelación espacial positiva, por lo que concluyen que dicho patrón tiene como consecuencia que las acciones anticorrupción de una nación pueden reducir la corrupción de sus vecinos. Además, los autores indican que el patrón de autocorrelación encontrado denota una cultura política regional. Por otro lado, se menciona que el grado de diseminación disminuye con la distancia geográfica a través de las regiones identificadas. 
Por su parte, Ortega, Florax y Delbecq (2010) encuentran que la corrupción presenta también un patrón de autocorrelación espacial positiva en una muestra de ciento cincuenta países. Las variables que incluyen como determinantes de la corrupción con mayor impacto son el producto por habitante y la libertad económica, lo que indica que el crecimiento de la economía hará crecer la corrupción, pero el aumento de la libertad económica tendrá el efecto de reducir la corrupción.

Donfouet, Wilter y Malin (2013), por su parte, construyen un modelo de panel espacial dinámico a nivel país que introduce la variable dependiente rezagada en el tiempo y en el espacio como determinante de la corrupción. Los autores reportan que esta es una función positiva tanto del espacio como de periodos anteriores. Dicho estudio toma como variable de observación el nivel de corrupción a nivel país con base en ciento cuatro observaciones. Los resultados indican que el producto por habitante y el nivel de libertad económica son variables de gran importancia en la modelación de la corrupción.

Pocos son los estudios que usan unidades de observación a nivel más desagregado. Del Monte y Paganini (2007), por ejemplo, llevan a cabo un análisis desagregado con veinte regiones en Italia en el periodo 1963-2000, y encuentran una relación positiva entre el producto por persona y la corrupción. Cabe señalar que este último estudio no presenta exploración espacial de la corrupción.

Diversos estudios hablan de la influencia que tiene el Gobierno sobre la corrupción. La teoría que sustenta esta relación se conoce como búsqueda de rentas, y según ella la expectativa de retornos altos o excesivos motiva el comportamiento económico. Cuando se parte de que la corrupción está ya instalada en una sociedad, la aplicación de la hipótesis de buscadores de rentas a servidores públicos indica que estos buscan obtener rentas, y la corrupción se convierte en una vía fácil de obtenerlas. Es decir, un mayor tamaño 
del Gobierno es un ambiente propicio para el surgimiento de la corrupción.

Los estudios existentes toman diversas variables para abordar la relación entre el tamaño del Gobierno y la corrupción. En este sentido Treisman (2000), Alí e Isse (2003) y Alesina y Angeletos (2005) encuentran que el tamaño del Estado está asociado linealmente con la corrupción. Así, de acuerdo con Campante y Quoc-Anh (2009), la corrupción disminuye con el tamaño del Estado en países en los que el gasto público es bajo, y la relación se mantiene positiva para rangos en los cuales el gasto de Gobierno es alto. En este mismo sentido, You (2005) indica que si favorece a los grandes conglomerados empresariales, la intervención del Estado en la economía puede incentivar la corrupción, y ofrece evidencia empírica para Corea.

Las variables demográficas reciben poca atención como determinantes de la corrupción. Una que sí resalta es el sexo. La literatura reporta estudios como el de Swamy, et al. (2000), quienes encuentran que las mujeres son menos propensas a caer en hechos corruptos identificables como soborno. Los autores atribuyen la menor propensión que tienen las mujeres a la corrupción a factores como la socialización, el acceso diferenciado a redes de corrupción y el menor acceso al empleo (y por ello, menor ingreso y menos contactos con la esfera pública). Otro resultado que va en este sentido es el reportado por Sung y Chu (2003), quienes hallan una relación negativa entre corrupción y tres niveles diferentes de medición de participación femenina.

\section{Corrupción y análisis espacial}

Un índice de percepción de la corrupción, llamado así por Transparencia Internacional y por los estudios mencionados, ha sido tradicionalmente usado para medir la corrupción al interior de un país. Este índice recibe amplia 
difusión internacional, tanto en la sociedad ${ }^{3}$ como en los medios de comunicación. El índice se publica anualmente para una gran cantidad de países alrededor del mundo desde mediados de los años noventa del siglo XX.

En dicho índice, México ha mostrado un deterioro muy grave en sus niveles de corrupción en los últimos años: pasó del lugar 75, en 2006, al lugar 123 en 2016. Para dimensionar esta caída se le puede comparar con Chile, que en el mismo periodo vio deteriorada de forma mínima su posición relativa, pues en el año 2006 ocupó el lugar 20 de la lista, y en el año 2016 ocupó la posición $24 .^{4}$

Los estudios sobre niveles de corrupción en épocas anteriores a los años noventa eran escasos. Uno de los pocos estudios al respecto es el de Mauro (1995), que construye índices de corrupción para cincuenta y cinco países con información del periodo 1980-1983. De acuerdo al índice de corrupción de este autor, México presentó, en esos años, los mayores niveles de corrupción entre los países latinoamericanos, ${ }^{5}$ sólo por encima de Haití; Chile, por su parte, era la nación con menores niveles de corrupción en el periodo mencionado.

Por su parte, la organización Transparencia Mexicana construye y publica el índice de corrupción y buen gobierno (ICYBG) a nivel entidad federativa. ${ }^{6}$ Este índice no es manejado como de percepción de la corrupción, a diferencia del que se publica a nivel país. De acuerdo con Transparencia Mexicana, el índice permite identificar, por un lado, los niveles de corrupción a nivel estatal y, por otro, el nivel de corrupción por cada tipo de trámite necesario para la obtención de un servicio público previamente definido

3.Aunque el índice es una aproximación, existen autores como Kauffman (1999) que confían en que la calidad de los índices puede soportar un análisis riguroso. 4. El país latinoamericano mejor posicionado en el ranking global de corrupción es Uruguay, que en 2016 ocupó el lugar 21.

5. Se incluyeron Chile, Brasil, Argentina, Colombia, Nicaragua, Panamá y Perú. 6. Los detalles de la construcción del índice pueden consultarse en:www.tm.org.mx 
(Transparencia Mexicana, 2003, p. 2). ${ }^{7}$ Además, cuantifica el número y el costo de los actos de corrupción.

La información de este último índice se obtuvo a partir de encuestas realizadas a jefes de hogar que utilizaron los servicios públicos mencionados a nivel entidad federativa. La unidad de registro se obtuvo cuando el entrevistado declaró haber pagado una suma de dinero a cambio de alguno de los servicios públicos considerados en la elaboración del índice.

Según Transparencia Mexicana, el ICYBG permite distinguir variaciones en niveles de corrupción de acuerdo con las características demográficas, sociales y económicas de la población. Los datos planteados en adelante en esta investigación han sido extraídos de este índice. La unidad territorial que contiene al fenómeno en cuestión son las entidades federativas del país, como en el ICYBG. La metodología llevada a cabo por Transparencia Mexicana indica que el tamaño de la muestra es suficiente para representar resultados a nivel de las entidades federativas.

La corrupción puede tomar varias dimensiones dependiendo del instrumento que se use para medirla. Un índice a nivel país no toma en cuenta las diferencias en los niveles de corrupción que puede haber en las diferentes zonas o regiones del país, pues generaliza los resultados. El ICYBG ancla el acto corrupto a la entidad federativa, en particular, en las dependencias de Gobierno en las que se dio dinero a cambio de la obtención del servicio. Esta manera de medir la corrupción hace que la medición de la misma esté bien enfocada y represente correctamente al fenómeno en términos territoriales. Obtener un índice de corrupción de una unidad más desagregada como el municipio, además de tener un alto costo, podría distorsionar los resultados pues no todos los servicios que se llevan a cabo a nivel entidad federativa

7. Para los índices del $200 \mathrm{I}$ y 2003 , se emplearon treinta y ocho servicios, y para 2005,2007 y 2010 , se usaron treinta y cinco servicios llevados a cabo en cada entidad federativa. 
se realizan a nivel municipal, como los trámites fiscales o aquellos en los que los habitantes de municipios alejados de ciudades deben trasladarse a estas para llevarlos a cabo, como el trámite de la obtención de pasaporte.

Cabe detenerse a reflexionar sobre la información del tipo de corrupción que es recabada por el índice. Existe corrupción en otros niveles de Gobierno, como federal o municipal, y en otras esferas del mismo, como en cámaras de diputados y senadores o en dependencias federales, que el índice no captura pues no está diseñado para ello. El ICYBG recoge la corrupción en la que participan la población y los funcionarios públicos respecto al cobro de servicios públicos, por lo que excluye otras formas y otros sectores de la economía. Es decir, la corrupción que se analiza no es toda la corrupción que hay en las entidades federativas.

Los valores que arroja el cálculo del ICYBG indican que sólo dos entidades federativas han estado por arriba de la media en todos los periodos: el Distrito Federal y el Estado de México, aunque Guerrero y Oaxaca son entidades que muestran altos niveles en el índice. Entre los años 2001 y 2010, el estado de Nayarit fue la única entidad que mostró una tendencia decreciente constante en el nivel de corrupción durante todo el periodo. Estados como Baja California Sur y Aguascalientes se encuentran por debajo de la media en todos los años.

De acuerdo con Donfouet, Wilter y Malin (2013), la relación funcional entre la corrupción de un estado y otro se debe, primero, a que las personas pueden moverse libremente al interior del territorio nacional y pasar de una región a otra e interactuar con las personas de esas entidades vecinas, por lo que los autores encuentran plausible responsabilizar a estas interacciones de las prácticas de corrupción. Segundo, en entidades federativas en las que hay Gobiernos que no fomentan el ataque a la corrupción o en las que las medidas para combatirla son tenues, la 
malversación de fondos y las transferencias ilegales pueden dirigirse a entidades federativas vecinas. Es por tales razones que se torna necesario aplicar técnicas de análisis espaciales para el fenómeno de la corrupción en México a nivel entidad federativa.

Los métodos de econometría espacial son utilizados primordialmente para identificar la existencia de dependencia espacial. ${ }^{8}$ El concepto de dependencia espacial según Anselin (1988) es "la existencia de una relación funcional entre un punto dado en el espacio y lo que ocurre en cualquier otro" (p. 11). La relación funcional mencionada hace referencia a que una variable localizada en un lugar puede impactar a la misma variable en otra localización. Para el caso de este trabajo, se busca probar que la corrupción de una demarcación impacta a la corrupción de otra demarcación.

Existen dos tipos de autocorrelación espacial: la positiva y la negativa. De acuerdo con Pérez (2006),

[...] la primera se presenta cuando la existencia de un fenómeno determinado en una región propicia su expansión a otras regiones circundantes y dicha expansión genera la concentración del mismo. De manera opuesta, la autocorrelación espacial negativa se refiere a la existencia de fenómenos en una región que impiden u obstaculizan la aparición de estos en otras regiones vecinas (p. 136).

Esta conceptualización resulta de gran importancia para este trabajo pues la identificación de un patrón de autocorrelación en la corrupción determinará que dicho fenómeno no se distribuya de manera aleatoria, sino que presente algún patrón de localización que manifestará en forma de aglomeración sobre el espacio. 
El primer paso en el análisis espacial es determinar si los datos ${ }^{9}$ presentan un patrón de autocorrelación espacial global. ${ }^{10}$ Para esto se utiliza el índice de Moran, que está definido como:

$$
I=\frac{N}{S_{0}} \frac{\sum_{i j}^{N} w_{i j}\left(x_{i}-\bar{x}\right)\left(x_{j}-\bar{x}\right)}{\sum_{i=1}^{N}\left(x_{i}-\bar{x}\right)^{2}}
$$

Donde $x_{i}$ es una variable en una región $i, x$ raya representa la media muestral de las observaciones en los $i$ territorios, $w_{i j}$ son los pesos de la matriz de proximidad $w, N$ es el tamaño de la muestra y $\mathrm{S}_{0}=\sum \sum w_{i j}$.

Este estadístico brinda una medida del grado de asociación lineal entre un vector de valores observados y el promedio ponderado de los valores de los vecinos de una observación. Los elementos de la matriz de proximidad $\left(w_{\mathrm{ij}}\right)$ toman valores unitarios cuando las zonas en cuestión comparten frontera, y cero en otro caso. Esta matriz será multiplicada por el vector de la variable que se pretenda someter a la exploración espacial para obtener un vector de valores ajustados por una matriz de pesos espaciales, conocido también como retardo espacial. Los elementos de la matriz $w$ resultan fundamentales debido a que estos recogen las interdependencias entre regiones. Si la I de Moran es positiva y significativa, implica la existencia de autocorrelación espacial positiva. Una I de Moran negativa y significativa implica autocorrelación espacial negativa. La matriz de pesos utilizada en este trabajo es una matriz tipo reina con una contigüidad de primer orden. ${ }^{11}$

9. Se le han aplicado logaritmos naturales a los valores del índice en todos los años. En adelante, se manejarán de esta manera.

I0. El ICYBG no se presenta con periodicidad anual, sino que está disponible para los años 200 I, 2003, 2005, 2007 y 20 I 0 . Es necesario decir que no hay publicaciones del índice en años anteriores ni posteriores.

II. Se obtuvieron los mismos resultados al hacer los cálculos con contigüidades de segundo y tercer orden. 
Tabla 1. Comportamiento espacial del ICYBG

$\begin{array}{cc}\text { Año } & \text { I de Moran } \\ 2001 & 0.1867^{*} \\ & (0.0420) \\ & 0.1702^{*} \\ 2003 & (0.0450) \\ & 0.1283 \\ 2005 & (0.0520) \\ & 0.2413^{*} \\ 2007 & (0.0170) \\ & 0.1793^{*} \\ 2010 & (0.0470)\end{array}$

*Estadísticamente significativo al 5\%

Fuente: elaboración propia.

Como se aprecia en la Tabla 1, el índice de Moran resulta positivo y estadísticamente significativo para todos los años, excepto para el 2005. El 2007 es el que resulta con mayor robustez, mientras que 2001, 2003 y 2010, aunque débiles, permiten sugerir la existencia de autocorrelación espacial positiva.

La diferencia en los valores del índice de Moran indica diversos grados de intensidad en la asociación espacial, significando que entidades con altos/bajos niveles de corrupción están rodeadas de entidades con altos/bajos niveles de corrupción. En otras palabras, existe un efecto de desbordamiento a través de las fronteras del ICYBG en todos los años, excepto el 2005. La intensidad del desbordamiento varía de acuerdo al valor reportado, es decir, el desbordamiento es mayor en 2007 y menor en 2001, 2003 y $2010 .{ }^{12}$

El caso del 2005 indica que en ese año el patrón de distribución fue aleatorio. Esto podría deberse al cambio de método empleado, pues Transparencia Internacional

12. Un ejemplo que puede servir de comparativo es el de Attila (2008), en el que los valores de la correlación espacial de la corrupción varín de un año a otro en cortes transversales. 
reporta que para los primeros dos años (2001 y 2003) la encuesta usada para obtener información incluyó treinta y ocho servicios, mientras que el año 2005 fue el primer año en que se descartaron siete servicios y se incluyeron cuatro nuevos, quedando treinta y cinco servicios incluidos en la encuesta, es decir, la metodología empleada cambió.

Usando las interpretaciones de Becker, Egger y Siedel (2009) y de Donfouet, Wilter y Malin (2013), el efecto de desbordamiento encontrado puede ser usado para describir una situación de contagio en los niveles de corrupción en las entidades federativas en los años en los que se mencionó que existe.

El siguiente paso en el análisis espacial es construir un indicador Local Indicator of Spacial Association (LISA), que se utiliza para buscar esquemas de dependencia en ciertas regiones, es decir, buscar autocorrelación espacial local:

El LISA descompone el patrón global de autocorrelación e indica en qué grado una región geográfica está rodeada por valores similares o disímiles formando un patrón regional. Los indicadores de LISA pueden integrarse en un mapa en el que se obtiene información sobre la significancia estadística de la aglomeración (Moreno y Vallá, 2000, p. 30).

Para el caso de la corrupción en México, el LISA es usado para identificar aglomeraciones (clusters) espaciales significativos alrededor de una entidad y localizar la región que contribuye en mayor medida al patrón de dependencia espacial en cada uno de los años de estudio. Cabe aclarar que los mapas que se pueden visualizar, una vez construido el LISA, indican la localización del centro del cluster.

El indicador LISA se presenta en mapas en los que las aglomeraciones tienen cuatro formas: alto-alto (high-high), cuando una entidad presenta altos niveles de la variable en cuestión y está rodeada de entidades con altos valores de dicha variable; alto-bajo (high-low), cuando una entidad 
presenta alto valor de la variable y se rodea de entidades con valores bajos de la misma; bajo-alto (low-high), cuando una entidad presenta valores bajos de una variable y está rodeada de entidades cuyo valor es alto; y bajo-bajo (lowlow), cuando una entidad tiene bajos valores de la variable y está rodeada de entidades con valores también bajos.

En lo subsecuente, se presenta el mapa de cluster de LISA. La lectura del Mapa 1, para el año 2001, indica que el cluster de regiones estadísticamente significativo se encuentra formado por el Estado de México, Guerrero y la Ciudad de México, mientras que las entidades que muestran significación estadística en bajos niveles de corrupción son Nuevo León y Baja California. El estado de Morelos muestra pertenencia al cuadrante de las entidades que tienen un indicador bajo del ICYBG rodeado de estados con altos niveles del mismo.

Para el caso del año 2003 el cluster que muestra significación estadística y que a su vez tiene los valores altos del ICYBG lo forman el Estado de México, Guerrero, Morelos, Puebla y Tlaxcala. La situación del estado de Morelos llama la atención pues dos años antes se encontraba entre las entidades de bajo nivel de corrupción, mientras que el cluster de valores bajos se mantiene en Baja California.

Para 2001, Oaxaca e Hidalgo son las entidades que tienen bajos valores del índice y se encuentran rodeadas de entidades con valores disímiles.

El mapa del año 2003 indica que el cluster de entidades con alta corrupción se encuentra formado por el Estado de México, la Ciudad de México y Tlaxcala.

Salta a la vista el estado de Guerrero, que en 2005 tiene bajos niveles del índice, pero que dos años antes se encontraba en la aglomeración de valores altos. 
Mapa 1. Mapa de cluster LISA 2001

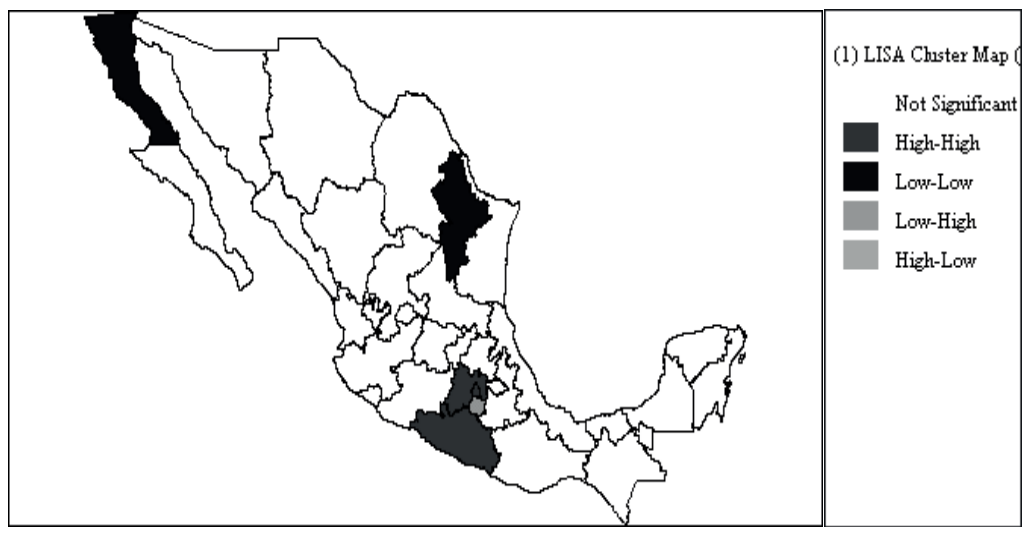

Fuente: elaboración propia con datos de Transparencia Mexicana (2001).

Mapa 2. Mapa de cluster LISA 2003

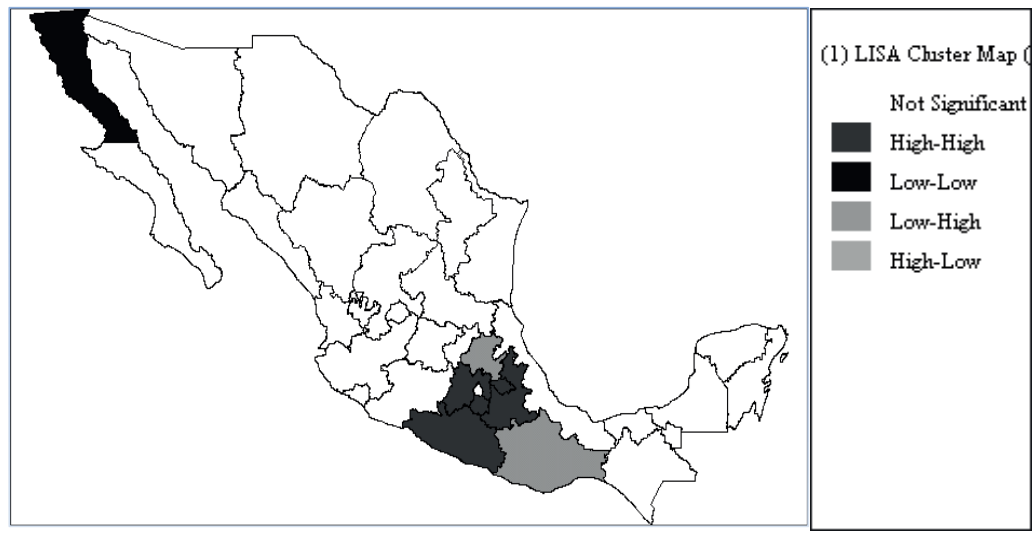

Fuente: elaboración propia con datos de Transparencia Mexicana (2003).

El mapa correspondiente al año 2007 indica que la aglomeración de altos valores del índice la forman el Estado de México, la Ciudad de México, Morelos, Puebla, Tlaxcala y Guerrero. 
El mapa del último año, 2010, indica que el cluster de bajos valores rodeado de entidades con altos valores lo forman los estados de Puebla, Morelos y Michoacán, mientras que el Estado de México presenta el alto valor.

Mapa 3. Mapa de cluster LISA 2005

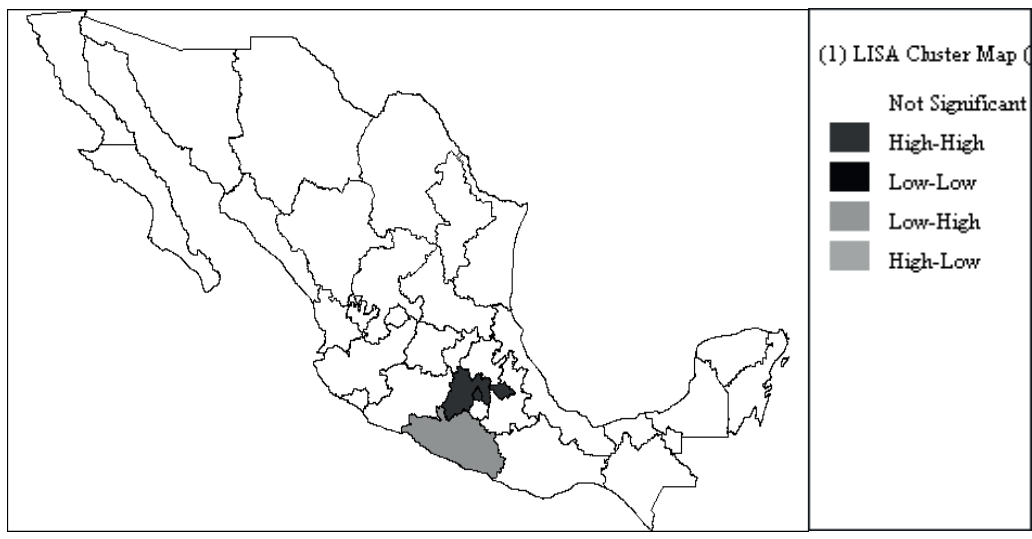

Fuente: elaboración propia con datos de Transparencia Mexicana (2005).

Mapa 4. Mapa de cluster LISA 2007

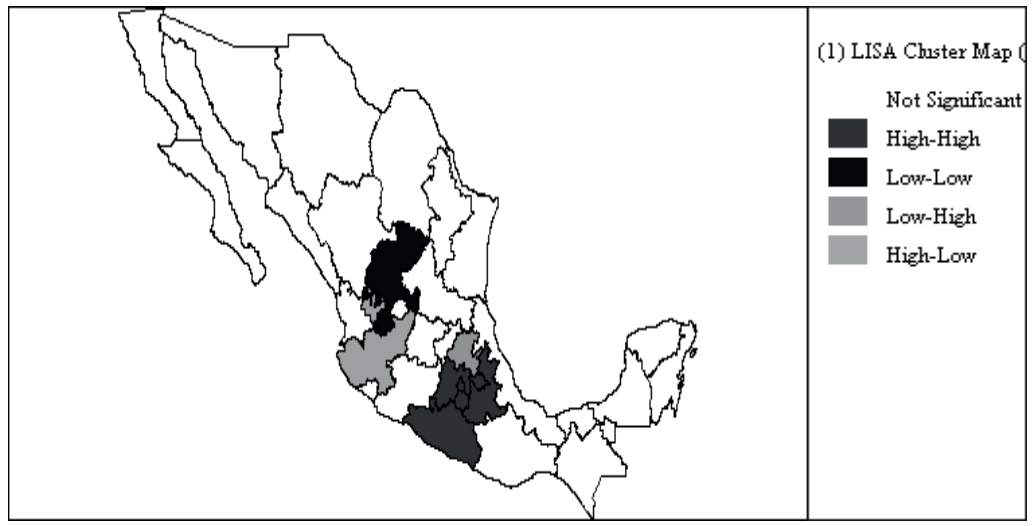

Fuente: elaboración propia con datos de Transparencia Mexicana (2007). 
Mapa 5. Mapa de cluster LISA 2010

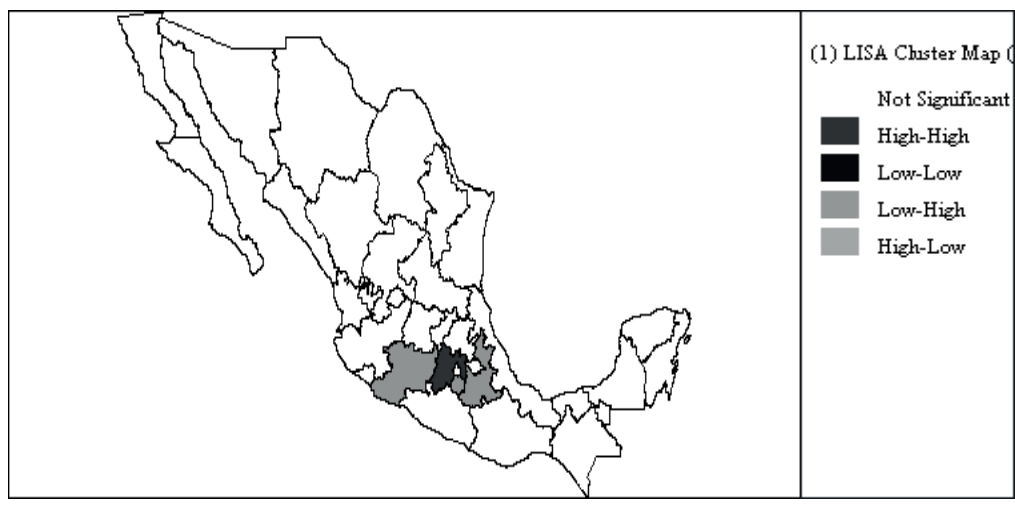

Fuente: elaboración propia con datos de Transparencia Mexicana (2010).

Llama la atención que el Estado de México es el único que es estadísticamente significativo en todos los años de estudio; esto lo caracteriza como el centro de la aglomeración de entidades con altos valores del ICYBG. También se ha podido observar que, en la mayoría de los años, las agrupaciones que muestran significancia estadística se localizan en el centro del país. Los pocos estados del norte del país que resultaron estadísticamente significativos pertenecieron al cluster de bajos niveles de corrupción.

\section{Análisis econométrico}

En este apartado se busca explicar el tipo de corrupción que se señaló anteriormente, es decir, la que relaciona a la población con servidores públicos. No se busca explicar la corrupción en general ni algún otro tipo o categoría. Además, los hallazgos posibles no se pueden extrapolar a otros tipos o formas de corrupción, pues estas pueden tener otros determinantes. 
Para modelar el ICYBG, se busca cuantificar el impacto que pueden tener la variable espacial, el producto por habitante y la intervención del Estado, así como el rol que juegan cada una de ellas en el índice. Estas variables han sido reportadas como determinantes del índice en la literatura especializada mencionada en el segundo apartado de este trabajo. Dada la complejidad del fenómeno, la literatura recomienda, en estudios como los de Ortega, Florax y Delbecq (2010), Ata y Arvas (2011) y Graff (2005), estudiar por separado los determinantes de la corrupción, dividiendo los determinantes económicos de los políticos y los socioculturales. No obstante, dada la importancia de las variables demográficas, se considera necesario tomarlas en cuenta. Para restringir lo menos posible los grados de libertad del modelo, se incluirá una variable de este tipo, que será el sexo. Como lo mostró la revisión de literatura, la inclusión de género está asociada con la corrupción.

Los resultados del modelo econométrico que se busca estimar ofrecerán un valor que, según Moreno y Vallá (2000), recoge la intensidad de las interdependencias entre las observaciones muestrales. El modelo a estimar se formaliza como:

$$
y=\rho W y+X \beta+\varepsilon
$$

Donde $y$ es un vector de datos de la variable dependiente, $W y$ es la variable dependiente espacialmente rezagada ajustada por una matriz de pesos espaciales $W, X$ es un matriz de observaciones de variables explicativas, $\mathcal{E}$ es un vector de errores independientes e idénticamente distribuidos, y $\rho$ y $\beta$ son coeficientes a estimar. Este modelo es conocido también como modelo mixto regresivo-autorregresivo espacial de primer orden. Para aplicar las matrices de contactos espaciales utilizadas en cada regresión, se tomó el criterio de Anselin (1988), según el cual se supone que no existe 
mecánica definida para elegir una matriz de este tipo, por ello, las matrices que se tomaron en cuenta fueron las que optimizaron la dependencia espacial (una matriz tipo reina con una contigüidad de primer orden). ${ }^{13}$ Cabe señalar que se aplicaron logaritmos naturales en todas las variables.

Se ha seguido el proceso reportado por Anselin (2005, p. 199) para determinar el modelo a estimar. El diagnóstico para la toma de decisión del modelo se presenta en la Tabla 2.

Tabla 2. Diagnóstico de dependencia espacial

\begin{tabular}{lcccccccccc} 
& \multicolumn{2}{c}{2001} & \multicolumn{2}{c}{2003} & \multicolumn{2}{c}{2005} & \multicolumn{2}{c}{2007} & \multicolumn{2}{c}{2010} \\
& Valor & Prob & Valor & Prob & Valor & Prob & Valor & Prob & Valor & Prob \\
LM & 5.53 & 0.01 & 1.18 & 0.31 & 0.68 & 0.40 & 4.90 & 0.02 & 2.35 & 0.12 \\
$\begin{array}{l}\text { (retardo) } \\
\text { LM robusta } \\
\text { (retardo) }\end{array}$ & 0.39 & 0.53 & 1.44 & 0.27 & 1.39 & 0.23 & 0.99 & 0.31 & 5.08 & 0.02 \\
$\begin{array}{l}\text { LM (error) } \\
\text { LM robusta } \\
\text { (error) }\end{array}$ & 0.40 & 0.05 & 0.41 & 0.51 & 0.37 & 0.54 & 4.04 & 0.04 & 0.43 & 0.51 \\
\hline
\end{tabular}

Fuente: elaboración propia.

Como puede apreciarse, el diagnóstico de dependencia espacial sugiere que sea estimado el modelo de rezago espacial para los años 2001, 2007 y 2010. La explicación de esto radica en que para estos años la asociación espacial del índice tomó un mayor valor que en los otros años, lo que indica que el efecto de desbordamiento se acentuó. La estimación de los modelos se puede observar en la Tabla 3. Cabe mencionar que para el año 2001 el PIB por habitante fue tomado con datos del Centro de Estudios de Finanzas Públicas (CEPF), mientras que para el resto de los años se utilizó el PIB calculado por el INEGI, pues el PIB por habitante no tuvo significación estadística. De la misma forma, la 
variable logaritmo del gasto de gobierno (LGG) fue probada en las regresiones como porcentaje del PIB y como gasto público por persona de cada entidad. No obstante, en ningún caso la LGG ofreció resultados estadísticamente significativos. Por todo esto, los resultados que se presentan fueron los obtenidos con el gasto público total.

La variable hombres fue tomada del Consejo Nacional de Población (CONAPO), y sólo fueron considerados los hombres mayores de edad. Se probó con la variable mujeres como porcentaje del total de habitantes por entidad federativa, pero se presentan los resultados de haber incluido hombres mayores de edad como proxy al sexo del jefe de familia, ${ }^{14}$ que fue quien respondió el cuestionario usado como fuente de datos, pues esta variable presenta mejor ajuste.

Tabla 3. Resultados de los modelos estimados

$\begin{array}{lccccc} & 2001 & 2003 & 2005 & 2007 & 2010 \\ \text { Constante } & -5.465 * & -0.756 & -0.396 & -1.355 & -2.871 \\ & (1.761) & (1.100) & (1.315) & (0.842) & (1.133) \\ \text { LPIB } & 0.259 * & 0.0004^{* *} & 0.394 & 0.4681^{*} & 0.416^{*} \\ & (0.128) & (0.000) & (0.274) & (0.136) & (0.228) \\ \text { LGG } & 0.457^{*} & 0.272^{*} & 80.034 & 0.1108 & 0.214 \\ & (0.090) & (0.123) & (0.165) & (0.093) & (0.131) \\ \text { HOM } & - & 0.036^{* *} & 0.075 & 0.032^{*} & - \\ & & (3.273) & (0.119) & (0.009) & \\ \text { Retardo espacial } & 0.421^{*} & - & - & 0.4009 * & 0.333^{* *} \\ & (0.158) & & & (0.1809) & (0.181) \\ \text { Pseudo - R } & 0.4722 & 0.313 & 0.100 & 0.459 & 0.3633 \\ \text { Breush-Pagan } & 0.1221 & 0.508 & 0.927 & 0.630 & 0.0701 \\ \text { p-valor } & & & & \\ \text { Error estándar entre paréntesis } & & & & \\ \text { *Significativo al 5\% } \\ \text { **Significativo al 1\% }\end{array}$

Fuente: elaboración propia. 
Las estimaciones presentadas indican que el PIB tiene impacto en el nivel de corrupción en cuatro de los cinco modelos. El coeficiente indica, en esos casos, una relación positiva entre el nivel de corrupción y el nivel de actividad económica. Esto significa que se cumple la hipótesis de Kindleberg, lo que coincide con los resultados encontrados por Paldam (2002), Braun y Di Tella (2004), Frechette (2006) y Ata y Arvas (2011).

El modelo de retardo espacial se estimó en tres de los cinco modelos. En ellos, el coeficiente resultó positivo y estadísticamente significativo, lo que coincide con los hallazgos de Becker, Egger y Siedel (2009) y Donfouet, Wilter y Malin (2013). El resultado indica que hay un desbordamiento en la corrupción entre las entidades federativas, excepto en los años en los que no se estimó el modelo. Comparativamente con estudios que han estimado coeficientes de retardo espacial en corrupción, se puede decir que el estimado en esta investigación es mayor, lo que indica que el efecto de desbordamiento entre entidades federativas ha tenido mayor intensidad en México.

Comparando los modelos entre los diferentes años, se puede apreciar que la variable de mayor impacto en el 2001 fue el tamaño del estado, seguido del retardo espacial. Una situación parecida se presenta en el modelo correspondiente a 2003. El año 2005 resulta muy particular, pues en él ninguna de las variables resulta significativa, lo cual genera la necesidad de buscar otras variables para modelar ese año. En el modelo del 2007, la variable de mayor impacto fue el PIB, seguido del retardo espacial, situación que se mantuvo en el 2010. Respecto al tamaño del Estado, los resultados permiten corroborar la hipótesis de búsqueda de renta sólo para los primeros años.

La variable porcentaje de hombres mayores de edad no fue incluida en los años 2001 y 2010, pues los resultados no fueron favorables para los objetivos de la investigación. En 
los años 2003 y 2007, la misma variable tuvo significación estadística y mostró signos positivos, sugiriendo que una mayor cantidad de hombres se asocia a un aumento de la corrupción.

La recomendación de políticas que se pueden derivar de este estudio puede resultar de gran importancia para combatir la corrupción desde un enfoque regional. El nivel de corrupción de los estados vecinos afecta el ICYBG a nivel entidad en casi todos los años de estudio. Esto significa que si en un estado el fenómeno incrementa, también lo hará en los estados contiguos. De la misma forma, el impacto de una política de combate a la corrupción que reduzca los niveles de esta en una entidad federativa tendrá el impacto de reducirla más allá de las fronteras de dicha entidad.

A la luz de este hallazgo, se puede argumentar que la política de combate a la corrupción a nivel entidad federativa debe tomar un enfoque suprarregional en el que se lleven a cabo acciones conjuntas y simultáneas para incidir sobre la entidad y sus vecinas. Incluso en el caso de los años en los que la corrupción no tenga asociación espacial, una política como la mencionada puede servir para prever su diseminación a través de las fronteras de las entidades federativas.

\section{Conclusiones}

Este trabajo exploró la distribución espacial a nivel entidad federativa del índice de corrupción y buen gobierno entre los años 2001-2010 en México. Los resultados muestran que la localización sobre el espacio del índice no es aleatoria, sino que muestra un patrón de autocorrelación espacial global positiva en cuatro de los cinco años de estudio. Esto significa que las entidades con altos/bajos niveles de corrupción se aglomeran con otras entidades de altos/ bajos niveles de corrupción. Este patrón había sido repor- 
tado a nivel país por autores como Becker, Egger y Siedel (2009) y Donfouet, Wilter y Malin (2013).

El análisis de los patrones de asociación local indica que la aglomeración de altos niveles de corrupción estadísticamente significativos se encuentra localizada al centro del país. La variable espacial, que es la de mayor interés para el presente trabajo, fue incluida en tres de los cinco años de estudio. El resultado indica una relación positiva y estadísticamente significativa, lo que sugiere la existencia de contagio de la corrupción entre entidades federativas con diferentes niveles de intensidad.

El nivel de producción mostró tener una relación positiva con la corrupción. Este resultado es coincidente con los hallazgos de Paldam (2002), Braun y Di Tella (2004), Frechette (2006) y Ata y Arvas (2011). De la misma forma, el tamaño del Estado se relacionó positivamente con la corrupción, pero este resultado sólo se pudo comprobar para dos años de los cinco estudiados.

Con base en los resultados, se recomienda combatir la corrupción tomando en cuenta las relaciones espaciales existentes entre entidades federativas. Es decir, el combate a la corrupción no debe llevarse a cabo suponiendo que los efectos de las acciones de política pública tendrán impacto solamente dentro de las fronteras de una entidad federativa, sino de manera regional, con la idea de que lo que suceda en una entidad afectará a lo que suceda en otra.

Alí, A., e lsse, H. (2003).“'Determinants of economic corrupBibliografía tion a cross country comparison”. Cato Journal, 22(3), 449-466.

Alesina, A., y Angeletos, G. (2005). “Corruption, Inequality and Fairness". Journal of Monetary Economics, 52, I227-1244. Aman Ullah, M., y Eatzaz, A. (2006). "Corruption and Income In-equality: A Panel Data Analysis" (ponencia 
presentada en el $22^{\text {nd }}$ Annual General Meeting of the Pakistan Society of Development). Islamabad, Pakistán.

Anselin, L. (1988). Spatial Econometrics Methods and Models. Dordrecht: Kluwer Academic.

(2005). Exploring Spatial Data with GeoDa: A Workbook. Estados Unidos: Center for Spatially Integrated Social Science.

Ata, Y., y Arvas, A. (20I I). "Determinants of economics corruption: A cross country data analysis". International Journal of Business and Social Sciences, 2(13), 161-169.

Attila, G. (2008). "Is corruption contagious? An econometrics analysis". NUPI Working Paper 742, I-33.

Becker, S., Egger, P., y Siedel, T. (2009). “Common Political Culture: Evidence on Regional Corruption Contagion". European Journal of Political Economy, 25(3), 300-3 I0.

Braun, M., y Di Tella, R. (2004). "Inflation, Inflation Variability and Corruption". Economics and Politics, (16), 77-100.

Campante, F., Chor, D., y Quoc-Anh, D. (2009). “Instability and the incentives for corruption". Economics and politics, 2 I (I), 42-92. Recuperado de: http://ink.library.smu.edu. sg/soe_research/409/

Cárdenas, J., y Mijangos, M. (2005). “Acerca del marco teórico de la corrupción”, en N. González Martín (coord.), Estudios Jurídicos en homenaje a Marta Morineau.Tomo II: sistemas jurídicos contemporáneos. Derecho comparado. Temas diversos (PP. 167-226). México: UNAM.

Casar, M. (2015). México: anatomía de la corrupción. México: Centro de Investigación y Docencia Económicas e Instituto Mexicano de la Competitividad.

CEEY (20I3). Informe de movilidad social en México. Recuperado de: https://www.ses.unam.mx/curso20 I3/pdf/ MovilidadSocialMexico.pdf

Chang, E., y Golden, M. (2007). "Electoral Systems, District Magnitude and Corruption”. British Journal of Political Science, 37(I), II5-I37. 
Chaudhry, A., y Shabbir, G. (2007). "Determinants of corruption in developing countries". Working Paper del Instituto de Economía Internacional de Hamburgo, (2-II). Recuperado de: https://www.econstor.eu/bitstream/I04 I 9/48268/I/6640 I365I.pdf

Del Monte, A., y Paganini, E. (2007). “The determinants of Corruption in Italy: Regional panel data analysis". European Journal of Political Economy, 23(2), 379-396.

Donfouet, H.,Wilter,J.,y Malin, E. (2013).“A Spatial Dynamic Panel Analysis of Corruption”. Working paper Centro de Investigación en Economía y Administración. Recuperado de: http://crem.univ-rennes I.fr/wp/20I3/20I324.pdf

Florino, N., y Galli, E. (20I0). "An analysis of the determinants of corruption: Evidence from Italian regions”. POLIS Working Paper, (I7I), I-27. Recuperado de: http://polis. unipmn.it/pubbl/RePEc/uca/ucapdv/fiorinol 7 I.pdf

Frechette, R. (2006). "A Panel Data Analysis of the TimeVarying Determinants of Corruption”. CIRANO Working Papers, (28), I-30. Recuperado de: http://www.cirano. qc.ca/pdf/publication/2006s-28.pdf

Goel, R., y Ram, R. (20/3). "Economic Uncertainty and Corruption: Evidence from a Large Cross Country Data Set”. Applied Economics, 45(24), 3462-3468.

Graff, J. (2005). "Consequences and causes of corruption: What do we know from a Cross-section of countries?". Working Paper University of Passau, 34(05), 4-35. Recuperado de: https://www.econstor.eu/bitstream/ I04 I 9/5503 I/I/684238772.pdf

Hodge, A., Shankar, S., Prasada, R., y Duhs, A. (2009). “Exploring the links between corruption and growth”. University of Queensland School of Economics Discussion Paper, (392), 2-57. Recuperado de: http://www.uq.edu.au/economics/ abstract/392.pdf

Huntington, S. (1972). El orden político en las sociedades en cambio. Buenos Aires: Paidós. 
Bibliografía

INEGI (20I4). Medición de la economía informal 20I 4. México: INEGI. Recuperado de: http://www.inegi.org.mx/est/ contenidos/proyectos/cn/informal/

Kauffman, D. (1999). "Governance Matters". World Bank Policy Research Working Paper, (2195), I-68. Recuperado de: http://documents.worldbank.org/curated/ en/66573 | 468739470954/pdf/multi-page.pdf

— y Wei, S. J. ( 1999). “Does 'Grease Money’ Speed up the Wheels of Commerce". NBER Working Paper, (7093), I-27. Recuperado de: http://www.nber.org/papers/ w7093.pdf

Kunicova, J., y Rose-Ackerman, S. (2005). "Electoral Rules and Constitutional Structures as Constrains on Corruption”. British Journal of Political Science, 34(4), 573-606.

Lederman, D., Loayza, N.,y Soares, R. (2005). “Accountability and Corruption:Political Institutions Matter". Economics and Politics, (17), I-35.

Lee III, R., y Thoumi, F. (1998). “El nexo entre las organizaciones criminales y la política en Colombia”. Ensayo y Error, (4). Recuperado de: https://es.scribd.com/document/ I 3 1998902/THOUMI-Francisco-El-nexo-entrelas-organizaciones-criminales-y-la-politica-en-Colombia Leff, N. (1964). "Economic Development through Bureaucratic Corruption”. The American Behavioral Scientist, (8), 8-I4.

Martínez, J. (2004). Conaliteg-Vamos México. Corrupción de Estado. El peón de la reina. Puebla: Benemérita Universidad Autónoma de Puebla.

Mauro, P. (1995).“Corruption and Growth”. Quarterly Journal of Economics, (I I0), 68I-7II2.

Moreno, R., y Vallá, E. (2000). Técnicas econométricas para el tratamiento de datos espaciales. Barcelona: Editions Universitat de Barcelona.

Ortega, D., Florax, R., y Delbecq, B. (20I0)."Primary determinants and the spatial distribution of corruption". 
Cuaderno de trabajo Universidad de Pardue, (10-6), I-I7. Recuperado de: http://ageconsearch.umn.edu/bitstream/I0I395/2/I0-6.pdf

Paldam, M. (2002). "The Cross-Country Pattern of Corruption: Economics, Culture and the Seesaw Dynamics". Journal of Political Economy, ( I8), 2I 5-240.

Pérez, J. (2006). "Econometría espacial y ciencia regional”. Investigación Económica, LXV, I29-160.

Serra, D. (2006). "Empirical determinants of corruption: A sensitivity analysis”. Public Choice, (I26), 225-256.

Soto, R. (2003). “La corrupción desde una perspectiva económica". Estudios Públicos, (89), 23-62.

Sung, H.E., y Chu, D. (2003). “Does participation in the global economy reduce political corruption? An empirical inquiry". International Journal of Comparative Criminology, 3(2), 94-I I8.

Swamy, A., Knack, S., Lee, Y., y Azfar, O. (2000). “Gender and Corruption”. CDE Working Paper Series. Recuperado de: http://web.williams.edu/Economics/wp/Swamy_gender. pdf

Thoumi, F. (1999). "La relación entre corrupción y narcotráfico: un análisis general y algunas referencias a Colombia". Revista de Economía de la Universidad del Rosario, 2(I), II-33.

Transparencia Internacional (2015). Corruption Perceptions Index. Recuperado de: https://www.transparency.org/ cpi20I5

Transparencia Mexicana (200I). Encuesta nacional de corrupción y buen gobierno 200I. Recuperado de: http://www. tm.org.mx/wp-content/uploads/2013/05/PresentacionEncuesta-Nacional-de-Corrupci\%C3\%B2n-y-BuenGobierno-200I.pdf (2003). Encuesta nacional de corrupción y buen gobierno 2003. Recuperado de: http://www.tm.org.mx/ 
Bibliografía

wp-content/uploads/20 I 3/05/Encuesta-Nacional-deCorrupcion-y-Buen-Gobierno 2003.pdf

(2005). Índice nacional de corrupción y buen gobierno 2005. Recuperado de: http://www.tm.org.mx/wp-content/uploads/20 I3/05/Informe-Ejecutivo-INCBG-2005. pdf

(2007). Índice nacional de corrupción y buen gobierno 2007. Recuperado de: http://www.tm.org.mx/wp-content/uploads/2013/05/Presentacion_INCBG_2007.pdf (20I0). Índice nacional de corrupción y buen gobierno 2010. Recuperado de: http://www.tm.org.mx/wpcontent/uploads/20 I3/05/0 I-INCBG-20 I0-InformeEjecutivol.pdf

Treisman, D. (2000). "The Causes of Corruption: A CrossNational Study”.Journal of Public Economics, (76), 399-457. You, J. S. (0I-04 de septiembre de 2005). "Embedded autonomy or crony capitalism? Explaining corruption in South Korea, relative to Taiwan and the Philippines, focusing on the role of land reform and industrial policy" (ponencia presentada en el Annual Meeting of the American Political Science Association). Washington, Estados Unidos. 